\title{
Unidades didácticas: Por una enseñanza asistida de la Matemática
}

\author{
Jairo José Flores Morales ${ }^{1}$ \\ Winston Joseph Zamora Díaz
}

\section{RESUMEN}

En forma progresiva, el aprendizaje de la matemática se ha mostrado como el problema díscolo del ámbito didáctico-pedagógico, claro está que esta difícil percepción recae sobre el actuar del estudiante, pero además sobre el quehacer del docente. Diversas investigaciones en esta disciplina han constatado que existen una serie de factores que dificultan el proceso de enseñanza- aprendizaje de la misma. En la búsqueda por dejar de suscitar problemas y dificultades ante esta compleja realidad, este ensayo recoge, desde el cuestionamiento y la reflexión aspectos vitales a tomar en cuenta a la hora de preparar y desarrollar nuestras prácticas docentes como profesores de matemática. A lo largo de las revisiones documentales y bibliográficas, y ante todo de la reflexión, lo que más destacamos es la necesidad de generar cambios en los procesos de enseñanza-aprendizajes de las matemáticas, esto desde un enfoque que fortalezca la contextualización de los contenidos y por ende la inmersión matemática.

Palabras clave: Enseñanza, matemática, didáctica, aprendizaje

Recibido: 15 de marzo de 2016

Aceptado: 10 de junio de 2016

1 Docente del Departamento de Ciencias de la Educación y Humanidades. Universidad Nacional Autónoma de Nicaragua, Managua. Correo Electrónico: jairofmdjmix@yahoo.com

2 Docente del Departamento de Ciencias de la Educación y Humanidades. Universidad Nacional Autónoma de Nicaragua, Managua. Correo Electrónico: winzamora@yahoo.es 


\title{
Didactic Units: Towards an assisted teaching of Mathematics
}

\begin{abstract}
The learning of the mathematics has proven to be a fractious problem in the didactical-pedagogical ambit. This perception depends in a high extent to the student's performance and the teacher's pedagogical work. Several studies have demonstrated the existence of a series of factors that difficult the teaching-learning process of this subject. This essay gathers vital aspects to take into account when preparing and developing our practices as mathematics teachers. Through document revision and reflection, we highlight the need to generate changes in the learning-teaching processes of the mathematics, this from an approach that empowers the contextualization of contents, and hence, the mathematical immersion.
\end{abstract}

Keywords: Teaching, mathematics, didactics, learning. 


\section{INTRODUCCIÓN}

La matemática se convierte en una ilusión de pocos y tortura de los demás cuando se hace ver como una asignatura fría, copada de fórmulas para resolver problemas extraídos de cualquier texto.

Oscar Villegas.

En forma progresiva, la enseñanza de la matemática ha venido sufriendo cambios radicales dentro del campo educativo. Diversas investigaciones en esta disciplina han constatado que existen una serie de factores que dificultan el proceso de enseñanza- aprendizaje de la misma.

Estos factores giran en torno a dos situaciones: Docente-Enseñanza, Alumno-Aprendizaje. Se ha llegado al punto de culpar a los docentes, alumnos, padres de familia, sistema de evaluación, metodología empleada, en fin, un sin número de culpables que lo único que ocasiona es ahondar la herida sangrante que replica en las expresiones de nuestros estudiantes: "Ésta asignatura no me gusta", "para qué me van a servir tantos contenidos", "Mejor ni estudio, pues ya estoy reprobado", "salgo mal porque no le entiendo a mi profesor", "me da clase de a chelín y me evalúa de a cinco pesos", "mi maestra no me motiva en clase". Estas realidades se pueden comparar con las siguientes pero desde el punto de vista magisterial: "el estudiante no estudia", "no tengo apoyo del padre de familia", "sale reprobado porque no pone atención en clase", "el sistema de educativo actual fomenta la pereza estudiantil", "antes se enseñaba mejor".

Obviamente, pareciera que ambos bandos fueran irreconciliables, pero gracias a diversos hallazgos investigativos, podemos afirmar que para cambiar esos esquemas mentales se necesita una ingeniería didáctica que facilite la enseñanza de la matemática. Ciertamente no es tarea fácil, en muchas ocasiones cuando un docente profundiza en un tema al principio solamente encuentra dificultades y caminos cerrados; sin embargo, se debe estar claro, que sin acciones concretas, sin publicar cuestiones que pongan de manifiesto la falta de compromiso docente y del sistema educativo; jamás se podrán mejorar los factores que envuelven negativamente tanto a docentes en la enseñanza, como a los estudiantes en su aprendizaje, y al sistema educativo mismo en la búsqueda de metodologías que propicien aprendizajes relevantes y pertinentes para la vida.

\section{DESARROLLO}

Teniendo en cuenta que la didáctica de la matemática se enfoca en la construcción de modelos teóricos para explicar los distintos aspectos de la enseñanza-aprendizaje de la matemática en el marco de los sistemas educativos, ésta no se puede desligar de los diversos problemas existentes en el campo de la enseñanza matemática, sin antes indagar el porqué de esos problemas y cómo darle un tratamiento idóneo. 
Actualmente, el primordial agente reflexivo de su propio quehacer es el docente, al respecto Godino (s.f) expone:

El mundo de la acción práctica es el campo propio del profesor, el cual tiene a su cargo uno o varios grupos de estudiantes a los cuales trata de enseñar matemáticas. El primer objetivo de un profesor es mejorar el aprendizaje de sus alumnos, de modo que estará principalmente interesado en la acción que pueda producir un efecto inmediato sobre su enseñanza.

Desde esta postura, la reflexión crítica en la acción puede afianzar, desarrollar o modificar el conocimiento profesional, para adaptarlo a las múltiples circunstancias concretas, que se dan en el proceso de enseñanza y aprendizaje. Uno de sus propósitos fundamentales es demostrar qué método de enseñanza es más efectivo.

El comprender la naturaleza del pensamiento del estudiante, sus motivaciones, intereses y sobre todo cómo aprende, deberían de ser algunas de las principales tareas del docente antes de iniciar a planificar la enseñanza y por otro lado, usar tales comprensiones para mejorar la educación en matemática.

Resultaría fantástico lograr que los estudiantes adquieran fascinación por la matemática y que a su vez lo conviertan en alumnos competentes, tal como lo define la Organización para la Cooperación y el Desarrollo Económico OCDE (2003), al decir que la competencia matemática es:

"La capacidad de un individuo para identificar y entender el papel que las Matemáticas tienen en el mundo, hacer juicios bien fundados y usar e implicarse con las Matemáticas en aquellos momentos en que se presenten necesidades en la vida de cada individuo como ciudadano constructivo, comprometido y reflexivo".

Por ende, se tiene que empezar a dar cambios radicales en nuestra forma de planificar nuestra labor docente. Ya no se puede seguir pensando que la mucha cientificidad que sabe el docente, le resultará sencillo enseñarlo. Acertadamente Díaz (1994), refiere que: "la peor expresión sería afirmar que si uno sabe bien un tema, le es posible enseñarlo; esta expresión es un rechazo cínico a la dimensión teórica de la educación”.

Consideramos que en Nicaragua, ha llegado el momento de darle importancia a propuestas didácticas que buscan fortalecer la enseñanza matemática. Todo esto, desde una perspectiva novedosa que busca minimizar los impactos desfavorables que ocasionan las prácticas docentes tradicionalistas y despreocupadas. 
No existe razón para no tratar vitales temáticas que fomentarían una lucha anuente por una enseñanza y aprendizaje de calidad, aspectos como:

- Fracasos en los intentos por desarrollar una apropiada comprensión de las matemáticas

- Contenidos desvinculados con el contexto en que vive el estudiante.

- El rol docente, continúa quedándose en el protagonismo tradicional durante el proceso de

- Enseñanza-Aprendizaje (E-A).

- La falta de sistematización y evaluación de la práctica docente en lo relacionado a la enseñanza de las matemáticas.

- El caso de las pruebas de ingreso a las Universidades como la Universidad Nacional Autónoma de Nicaragua (UNAN-Managua) y la Universidad Nacional de Ingeniería (UNI).

- Poca utilización de herramientas tecnológicas en la enseñanza de matemática.

- Disposición al cambio por parte del profesorado.

De ahí surgen algunas preguntas que como docentes de matemática nos debemos hacer: ¿Cuál ha sido mi roll ante éstas problemáticas?, ¿Por qué sólo se consiguen aprendizajes en aquellos estudiantes con potencialidades para aprender y en los otros casos no se logra el aprendizaje aunque el profesor sea competente?, ¿Qué debemos hacer para enfrentar y resolver ésta situación?, ¿cómo planificar la docencia?, y una de las más importantes, ¿Estoy dispuesto a utilizar la didáctica como eje fundamental para lograr una enseñanza asistida de la matemática?

Cada interrogante se puede responder si nuestra docencia la convertimos en un acto constante de evaluación. Evaluación que serviría para re direccionar la práctica docente y mejorar la planificación didáctica enfocadas a entender cómo aprenden nuestros estudiantes.

Acertadamente Pérez (2008), plantea la necesidad de analizar las vías fundamentales por las que los seres humanos adquieren significados (aprenden), ya que esto constituye un referente obligado para el desarrollo competente del quehacer docente. Es decir, es necesario tener claro cómo aprende el alumno, ya que la tarea del profesor será la de apoyar estas formas de aprendizaje. Todo esto es posible mediante una acción que es inherente al rol docente y que todos conocemos como planificación.

Zabalza (2009), en su libro Competencias docentes del profesorado universitario señala que planificar el proceso de enseñanza-aprendizaje es la competencia con la que debe despuntar quien asume el rol de profesor universitario. Es por el hecho de que la planificación es lo que concibe su actuación, el tratamiento de los contenidos, la contextualización de los mismos y el curso como un proyecto de desarrollo.

A través de la planificación es posible enfrentarse al control de una serie de variables a las que el profesorado se enfrenta durante el desarrollo de la práctica educativa. Todo lo relacionado con la planificación debe quedar concretado a manera de una documentación. Pero además de pensar en los contenidos temáticos a transmitir, debemos tomar en cuenta los siguientes cuestionamientos: 
¿Cómo conocer el nivel de conocimiento de mis estudiantes?, ¿Qué deben aprender hoy mis estudiantes?, ¿Para que deben aprenderlo?, ¿Con qué medios o recursos puedo lograr esos aprendizajes?, ¿Cómo puedo fomentar/provocar los aprendizajes? ¿Con qué actividades?, ¿Cómo puedo junto con mis estudiantes reconocer su progreso?, ¿Cómo puedo saber si mi actuar docente ha ayudado o perjudicado? ¿Cómo evaluar realmente? y ¿está bien lo que evalúo?

Es por eso que para conseguir lo antes expresado sobre la práctica educativa es ineludible realizar la planificación del acto enseñante. Para ello es necesario apropiarse de una de las tantas formas. Llega el momento de decidir de qué manera o en que formato quedará mejor reflejada todas las cuestiones que implica esa planificación. Después de haber realizado diversos trabajos investigativos en didáctica de la matemática, proponemos una planificación por medio de unidades didácticas.

Las unidades didácticas son una forma de programación o planificación de la enseñanza en un tiempo determinado, en la misma se pretende incluir al máximo los elementos que intervienen en el proceso de enseñanza-aprendizaje.

Podemos afirmar, que la unidad didáctica es la interrelación de todos los elementos que intervienen en el proceso de enseñanza-aprendizaje con una coherencia interna metodológica y por un periodo de tiempo determinado. Algunas de sus principales ventajas son:

- Puede abarcar el tratamiento de varios contenidos

- Permite relacionar la teoría con la práctica.

- Orienta el actuar del docente y del estudiante en cada momento.

- Evita el papel monólogo del docente.

- Como alternativa es propio de la metodología constructivista.

- Contiene el qué, el para qué y el cómo del desarrollo de la clase.

- Orienta el proceso de evaluación en todo momento.

Entre sus elementos tenemos la descripción de la unidad didáctica, los objetivos didácticos, contenidos de aprendizaje, secuencia de actividades, recursos materiales, organización del espacio y tiempo, y la evaluación. Cada uno de estos elementos cumple con los estándares que actualmente se exigen en los distintos niveles de educación nicaragüense.

Con esta forma de planificar, el docente presta atención a la organización de la enseñanza y el aprendizaje, lo que los alumnos aprenden y fundamentalmente de cómo se lleva a cabo este aprendizaje (Godino, 2004), por otro lado, el contexto donde se desarrolla dicho proceso evidencia una ruptura clara de paradigmas que no favorecen en lo absoluto a la enseñanza matemática como tal. 
Se hace evidente, que una planificación inadecuada desfavorece la obtención de competencias, más aún, si las actividades que se presentan son una repetición que el estudiante realiza en las actividades ejemplificadas por su maestro, de ahí la importancia de las unidades didáctica y alfabetizar al estudiantado en matemática de una forma diferente. Eficazmente (Rico, 2006: 276277) expresa:

"Alfabetización o competencia matemática general se refiere a las capacidades de los estudiantes para analizar, razonar y comunicar eficazmente cuando enuncian, formulan y resuelven problemas matemáticos en una variedad de dominios y situaciones. Un buen nivel en el desempeño de estas capacidades muestra que un estudiante está matemáticamente alfabetizado o letrado, reducir la noción de alfabetización a sus aspectos instrumentales más básicos, al simple dominio de conceptos y técnicas, puede resultar excesivamente elemental".

De ahí la importancia de una planificación innovadora que logre alcanzar dichas competencias, si nos situamos en nivel de planificación del docente, las componentes en las que se concreta el currículo en cada una de sus dimensiones son los contenidos, los objetivos, la metodología y la evaluación; el modelo de los organizadores del currículo le suministra una serie de herramientas de cara al diseño de actividades de enseñanza y aprendizaje (Lupiáñez, 2009).

En las unidades didácticas, se debe realizar una cuidadosa selección de las tareas y situaciones didácticas que proporcionen oportunidades a los alumnos para indagar problemas significativos para ellos y sobre todo relevantes desde el punto de vista matemático, formular hipótesis y conjeturas, utilizar diversos tipos de representaciones; realizar buenas preguntas, validar sus soluciones y comunicarlas a otros, dentro de un clima cooperativo y científico, todo esto permite que la interrelación entre los diferentes contenidos sea máxima (Zabala , 2008). Razón fundamental para usar contratos didácticos que proporcionen responsabilidad tanto del docente, como del estudiante.

Los estudiantes aprenden con tareas presentadas en forma de juegos, aprovechando la tendencia natural que tienen a formar grupos, utilizando el aprendizaje cooperativo como estrategia de atención a la diversidad, fomentando la metacognición, e incluso a los problemas matemáticos, convenientemente escogidos y dosificados, pueden ser muy útiles para el desarrollo del pensamiento matemático, y a la vez, ampliar las estrategias apropiadas en los estudiantes para cada situación conflictiva (Alcina et al,2004). Esta forma de planificación permite, aprender de forma comprensiva, potenciar la explicitación de ideas, conocer las características y habilidades de los alumnos y valorar los errores de los estudiantes como elemento indispensable para enrumbar los aprendizajes (Dolors y Montserrat, 2008).

Cabe señalar, que el modelo evaluativo de esta forma de planificación rompe el esquema que lamentablemente aún perdura en nuestros días, al evaluar exclusivamente resultados, obviando 
todo el proceso. La aplicación de una forma de evaluar que regule los saberes de los estudiantes, permite que las actividades plasmadas en la unidad didáctica sirvan en primera instancia para obtener aprendizajes competenciales, pero a la vez, para autorregular el propio aprendizaje obtenido por el estudiante.

Aspectos como los señalados anteriormente, son los que hacen de las unidades didácticas una propuesta alternativa que apunta hacia una enseñanza asistida de las matemáticas en cualquier nivel escolar.

\section{CONCLUSIONES}

Con el deseo incansable de incitar a la reflexión, y luego de haber discurrido sobre una problemática latente, de largo recorrido, pero de menospreciada atención, esperamos cerrar este ensayo, pero estimular apenas a un diálogo conjunto encaminado hacia propuestas alternativas que puedan abrir camino hacia una inmersión de las matemáticas. A continuación presentamos a manera de conclusión aspectos que induzcan hacia una reflexión crítica, y que aunque no son la panacea, consideramos merecen atención.

- Tradicionalmente hemos enseñado matemáticas expresándole a nuestros estudiantes que sirven para todo, pero muy pocos o casi ninguno de nosotros (profesores) las hemos utilizado para sobresalir en algún evento científico, para promover la innovación, y a veces ni para resolver acciones cotidianas, no más hacemos docencia. Debemos reconocer que las matemáticas seguirán siendo una ciencia integral y un complemento fundamental para el desarrollo de la ciencia y la tecnología, pero eso no lo veremos concretar en nuestro entorno mientras sigamos aprendiendo y enseñando matemáticas sin justificar su historia, el porqué, el cómo aplicarlas y para qué. Estos elementos que predominan con carencia en este proceso son los que permitirían la mencionada motivación.

- Debemos evidenciar la necesidad de generar cambios en los procesos de enseñanza-aprendizajes de las matemáticas, esto desde un enfoque que fortalezca la contextualización de los contenidos y por ende la inmersión matemática.

- Asumir con ahínco que la labor docente frente al desafío de aprender y enseñar matemáticas no es una actividad de abordaje sencillo, por lo que requiere de mucho empeño, de formación permanente y dinámica.

- Debe ser una prioridad desde los distintos subsistemas, principalmente en la Universidad, promover y desarrollar prácticas didácticas que estimulen la investigación matemática, la incorporación de las TIC's como un elemento que permita experiencias manipulativas, creativas y extrapolables a situaciones del contexto, eludiendo enfrascarse en los planteamientos academicistas-bibliográficos que niegan concretamente la motivación por aprender matemáticas, y por tanto, la trascendencia de ésta.

- Alertar a las Universidades encargadas de formar profesores de matemáticas, que éstos requieren una formación que estimule la investigación matemática, y les encamine por una 
didáctica de las matemáticas que incite hacia formas dinámicas y creativas de planificación.

- Es necesario ahondar en una planificación y abordaje de las matemáticas que estimule a la comunicación de los aprendizajes, al cálculo, a analizar, a la interpretación, al optimizar, al demostrar, a comparar, a gestionar, en fin a acciones que conlleven a que las matemáticas tengan más sentido fuera del aula de clase.

- Fortalecer la visión formadora del profesorado de matemáticas por comprender que es necesario entender la epistemología matemática, pero que es pertinente también comprender y dar respuesta a los distintos ritmos de aprendizajes, a la gestión del conocimiento, a la convivencia en el aula; en fin todo lo implicado al ambiente escolar como espacio tradicional donde acontece el aprendizaje.

\section{BIBLIOGRAFÍA}

Alcina, C. Alcalá, M. Aldana, J. Bishop, A. Carbó, L. Colomer, T. Fernández, A. Ferrero, L. García, A. Giménez, J. Hans, J. Monterde, M. Mora, J. Muñoz, J. Pazos, M. Ramos, N. Recarens, E. y Segarra, L. (2004). "Matemáticas re-creativas”. Barcelona: Graó;20-23.

Díaz, A. (1994). "Currículo y tecnología educativa”. Disponible en: http://goo.gl/xvi64C

Dolors, A. y Montserrat, V. (2008). “Juegos de expresión oral y escrita”. Barcelona: Graó; 5-6.

Godino, J. (2004). "Didáctica de las matemáticas para maestros”. Granada: Gami, 91-95;425-456.

Godino, J. (s.f). Presente y futuro de la investigación en didáctica de las matemáticas. Disponible en: http://goo.gl/37Rvoi

Lupiáñez, J. (2009). "Expectativas de aprendizaje y planificación curricular en un programa de formación inicial de profesores de matemáticas de secundaria”. Obtenida el 14 de febrero de 2013, de http://goo.gl/q6gMh

OCDE. (2003). "El marco de evaluación de PISA 2003; Matemáticas, lectura y resolución de problemas del conocimientos y habilidades". Paris. OCDE

Pérez, A. (2008). ¿Competencias o pensamiento práctico? La construcción de los significados de representación y de acción. En: J. Gimeno-Sacristán (Comp.). Educar por competencias, ¿qué hay de Nuevo? Madrid: Morata, pp. 59-102.

Rico, L. (2006). Marco teórico de evaluación en PISA sobre matemática y resolución de problemas. [Versión electrónica].Revista de educación.(Número extraordinario 2006), 275-294.

Zabala, A. (2008). “La práctica educativa. Cómo enseñar”. Barcelona: Graó; 35-47,179-181.

Zabalza, A. (2009). Competencias docentes del profesorado universitario, calidad y desarrollo professional. Madrid: NARCEA. 